\title{
Scaling properties of weakly nonlinear coefficients in the Faraday problem
}

\author{
A. C. Skeldon* \\ Department of Mathematics, Faculty of Engineering and Physical Sciences, University of Surrey, Surrey GU2 7XH, United Kingdom
}

J. Porter

E.T.S.I. Aeronáuticos, Universidad Politécnica de Madrid, Plaza Cardenal Cisneros 3, E-28040 Madrid, Spain

(Received 22 December 2010; revised manuscript received 19 April 2011; published 18 July 2011)

\begin{abstract}
Interesting and exotic surface wave patterns have regularly been observed in the Faraday experiment. Although symmetry arguments provide a qualitative explanation for the selection of some of these patterns (e.g., superlattices), quantitative analysis is hindered by mathematical difficulties inherent in a time-dependent, free-boundary Navier-Stokes problem. More tractable low viscosity approximations are available, but these do not necessarily capture the moderate viscosity regime of the most interesting experiments. Here we focus on weakly nonlinear behavior and compare the scaling results derived from symmetry arguments in the low viscosity limit with the computed coefficients of appropriate amplitude equations using both the full Navier-Stokes equations and a reduced set of partial differential equations due to Zhang and Vinãls. We find the range of viscosities over which one can expect "low viscosity" theories to hold. We also find that there is an optimal viscosity range for locating superlattice patterns experimentally-large enough that the region of parameters giving stable patterns is not impracticably small, yet not so large that crucial resonance effects are washed out. These results help explain some of the discrepancies between theory and experiment.
\end{abstract}

DOI: 10.1103/PhysRevE.84.016209 PACS number(s): 05.45.-a, 47.20.Ky, 47.35.-i, 47.54.-r

\section{INTRODUCTION}

Numerous instabilities in physics, chemistry, and biology can trigger a transition from a homogeneous state to a spatially patterned one. These patterned states can be regular or irregular, steady or periodic, and much effort has gone into understanding the factors that determine their characteristics. In particular, there is an important distinction between those aspects of the pattern that depend on the detailed physical properties of the system, and those that result more generally from its symmetries. One of the systems that has proven most fruitful in illuminating this distinction, and pattern formation in general, is the Faraday system [1]-in its most common configuration, an open container of fluid shaken up and down to provoke surface waves. This system is relatively compact, evolves on a convenient time scale, and is conducive to simple controlled experiments. At the same time, it can produce a tremendous variety of interesting patterns, depending on the applied forcing and fluid properties.

Experiments on the Faraday system may be loosely divided into two categories: those using low viscosity fluids such as water (kinematic viscosity of about $1 \mathrm{cSt}$ ) with single-frequency harmonic excitation [2,3], and those using moderate to high viscosity fluids such as silicone oil (typically with kinematic viscosity of $10 \mathrm{cSt}$ or greater) with multifrequency excitation [4-8] composed of several rationally related frequency components $\{m \omega, n \omega, p \omega, \ldots\}$ with $\{m, n, p, \ldots\} \in \mathbb{Z}$. Low viscosity experiments are characterized by a large correlation length, making them sensitive to boundary conditions and container shape, while more viscous fluids are less affected by the container boundaries, and are thus more representative of the intrinsic pattern formation properties of the infinite system. Proximity to the infinitely extended limit, which possesses

\footnotetext{
*a.skeldon@ surrey.ac.uk
}

the full Euclidean symmetry of the plane, is an advantage theoretically due to the simplicity of the eigenfunctions (the spatial part can be decomposed into Fourier modes) and the constraints that Euclidean symmetry imposes on their interactions.

Symmetry constraints are manifest in the form of the amplitude equations describing the weakly nonlinear interactions of excited (critical) modes near onset. The fact that certain terms are prohibited in these amplitude equations, along with the coefficient values of the allowed terms, largely determine the observed patterns $[9,10]$. It is generally necessary to perform a lengthy calculation to obtain these important nonlinear coefficients. In the limit of low viscosity, however, the residual effects of weakly broken Hamiltonian and temporal symmetries can be exploited to obtain various predictions about the scaling and phase dependence of these coefficients without the need for explicit calculations [11,12]. These predictions suggest ways that pattern selection might be "controlled" through judicious choice of the components in a multiple-frequency excitation [12].

Many of the conclusions of this symmetry-based theory have been tested against explicit amplitude equations calculated from a model partial differential equation derived from the Navier-Stokes (NS) equations by Zhang and Vinãls (ZV) [13], and have shown excellent agreement. However, the derivation of the $\mathrm{ZV}$ model from the NS equations relies on the assumptions of low viscosity and infinite depth. The experimental results on multifrequency forced pattern formation use fluids of moderate to large viscosity. Consequently, even though the theory is in good agreement with the $\mathrm{ZV}$ model, the extent to which that theory and the $\mathrm{ZV}$ model itself can be applied to the pattern formation experiments of interest remains unclear.

Recently, a method for performing the weakly nonlinear analysis for multifrequency forced Faraday waves for the NS 
equations has been developed, enabling the coefficients of the amplitude equations to be derived [14] without recourse to the $\mathrm{ZV}$ model. The purpose of this paper is to compare results obtained with this method from the full NS equations, avoiding the assumption of low viscosity, with the scaling laws given in $[11,12]$ and with the corresponding results from the $\mathrm{ZV}$ model. We thus investigate the range of validity of recent symmetry-based predictions, as well as the ZV model itself, as damping is increased.

The paper is organized as follows. In Sec. II, relevant background on the linear stability problem and on the mechanisms of nonlinear pattern selection is provided. In Sec. III, a detailed comparison of the NS model, the ZV model, and symmetry-based predictions is made. This includes, for two-frequency excitation, a comparison of cubic normal form coefficients in Sec. III A, a discussion of different methods for calculating the resonant angle in Sec. III B, and a comparison of quadratic normal form coefficients in Sec. III C. In Sec. III D, the cubic normal form coefficients corresponding to three-frequency experiments are investigated. In Sec. III E, the implications of the scaling behavior of important normal form coefficients for experimental pattern observation of superlattice and quasipatterns are discussed. Lastly, in Sec. IV we present our conclusions.

\section{MECHANISMS OF PATTERN SELECTION}

\section{A. Linear problem}

In the Faraday system, considerable insight into wave pattern selection can be obtained directly from the linear stability problem. Benjamin and Ursell [15] showed that in the inviscid, infinite depth limit the linearized Faraday problem reduces to a Mathieu equation, meaning that the instability boundaries organize into resonance tongues associated with half-integer multiples of the fundamental forcing frequency. In the ZV model, which assumes weak viscosity, the linear problem is instead described by a damped Mathieu equation: The instability boundaries of the flat surface solution form a series of resonance tongues, the lowest of which indicates the (first) critical mode. This same basic structure for the linear stability problem carries over to the full NS problem.

With single-frequency forcing of the form $f(t)=a \cos (\omega t)$ the first instability is nearly always to the primary subharmonic mode associated with the frequency $\omega / 2$, but with multifrequency forcing the situation can be considerably more complex due to the selective excitation of other resonance tongues. In this case, the first instability is generally not to the first subharmonic mode, but to a mode associated with the frequency $m \omega / 2$, where $m \omega$ is the "dominant" component (due to the variation of damping with frequency, this does not necessarily mean that it is the largest forcing component). In this more general context, one refers to a mode as subharmonic (harmonic) if $m$ is odd (even), meaning it oscillates through an odd (even) number of half-periods during one period of the forcing. This odd or even character of the modes under discrete time translation symmetry $t \rightarrow t+2 \pi / \omega$ is essential in determining the form of the amplitude equations [10], implying, for example, that quadratic self-interaction terms can only occur for harmonic modes.
In general, increasing the excitation amplitude $a$ leads to patterns only beyond a critical value $a_{c}$ given by the minimum of the lowest instability tongue, whose position determines the critical wave number $k_{c}$. For low viscosity fluids, an estimate for $k_{c}$ can be obtained from the dispersion relation for gravitycapillary waves in an inviscid fluid

$$
\Omega^{2}=g k+\frac{\sigma}{\rho} k^{3}
$$

where $g$ is the acceleration due to gravity, $\sigma$ the surface tension, and $\rho$ the density. In this case the solution $k=k_{0}$ of Eq. (1) with $\Omega=m \omega / 2$ is approximately equal to $k_{c}$. We take $1 / k_{0}$ as the characteristic length for the problem and $1 /(m \omega)$ as the characteristic time, meaning that the dimensionless critical wave number is near unity while the nondimensional period of the critical mode is $4 \pi$. This differs from the usual choice of $1 / \omega$ [16] for characteristic time, but it facilitates comparison between different multifrequency forcing functions because it is less sensitive to small changes in their content; patterns induced by frequency components at $50 \mathrm{~Hz}$ and $100 \mathrm{~Hz}$, for example, do not require a drastically different scaling from those with frequency components $51 \mathrm{~Hz}$ and $100 \mathrm{~Hz}$. In nondimensional form Eq. (1) becomes

$$
\tilde{\Omega}^{2}=G_{0} \tilde{k}+\Gamma_{0} \tilde{k}^{3},
$$

where $G_{0}=g k_{0} / \omega_{c}^{2}, \Gamma_{0}=\sigma k_{0}^{3} /\left(\rho \omega_{c}^{2}\right), \tilde{\Omega}=\Omega / \omega_{c}, \tilde{k}=k / k_{0}$, and $\omega_{c}=m \omega$ is the dominant frequency ( $m=1$ for singlefrequency forcing); note that the definition of $k_{0}$ requires that $G_{0}+\Gamma_{0}=1 / 4$. We further introduce the dimensionless damping parameter $\gamma=2 v k_{0}^{2} / \omega_{c}$, where $v$ is the kinematic viscosity.

As an example, consider the applied acceleration,

$$
f(t)=f_{m} \cos \left(m \omega t+\phi_{m}\right)+f_{n} \cos \left(n \omega t+\phi_{n}\right),
$$

with $m: n=6: 7$, and

$$
\left(f_{m}, f_{n}\right)=a G_{0}(\cos \chi, \sin \chi) .
$$

The mixing angle $\chi$ provides a convenient way of varying the proportions of $f_{m}$ and $f_{n}$ while keeping the dimensionless amplitude $a$ (measured in units of $g$ ) constant. The critical curves for three different values of $\chi$, computed from the infinite depth NS equations using the method of [17], are shown in Fig. 1.

When $\chi=0^{\circ}$ [Fig. 1(a)], the unique forcing frequency is $6 \omega$, and the lowest (and largest) resonance tongue is associated with $\Omega=3 \omega$. This is just the usual single-frequency case, dominated by the first subharmonic instability tongue. In the original two-frequency context, however, the mode is considered harmonic because its dominant frequency is an integer multiple of $\omega$. When $\chi=90^{\circ}$ [Fig. 1(c)], the unique forcing frequency is $7 \omega$, and the single-frequency picture applies again. Note that, for the sake of comparison, in this particular case we have retained the scaling associated with a dominant $6 \omega$ component: If scaling in accord with $7 \omega$ forcing had been used the main tongue would be closely centered on $k=1$, as in Fig. 1(a). This time the critical mode is subharmonic even in the two-frequency context because its dominant frequency is $\Omega=3 \omega / 2$, a half-integer multiple of $\omega$. In both these cases there are additional tongues for higher values of $k$, but these are not visible on the scale of the plot. The 
(a)

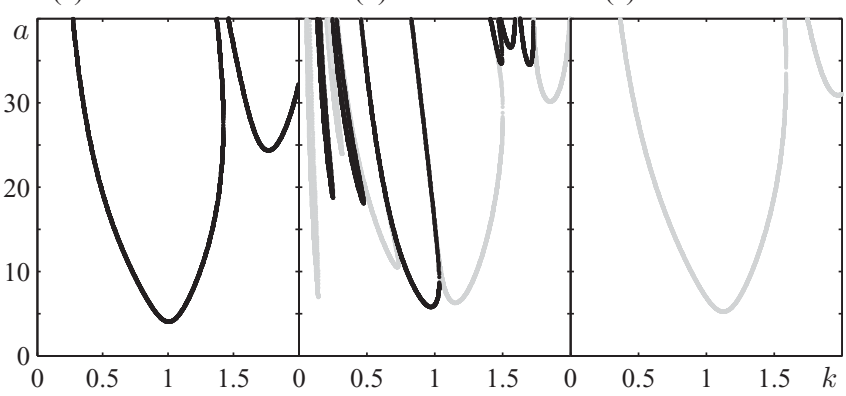

FIG. 1. Linear stability curves for the NS equations for a twofrequency component forcing of the form (3) with $m: n=6: 7$, $\phi_{m}=\phi_{n}=0$ and (a) $\chi=0^{\circ}$, (b) $\chi=55^{\circ}$, (c) $\chi=90^{\circ}$. Remaining parameters are $\rho=0.95 \mathrm{~g} / \mathrm{cm}^{3}, g=980 \mathrm{~cm} / \mathrm{s}^{2}, \sigma=20.6 \mathrm{dyn} / \mathrm{cm}$, $\nu=12.89 \mathrm{cSt}$, and $\omega=32.88 \pi$, which correspond to $G_{0}=0.0396$, $\Gamma_{0}=0.2104$, and $\gamma=0.1$. The black (gray) curves represent harmonic (subharmonic) instabilities.

most interesting parameter range is near $\chi=55^{\circ}$ where the $6 \omega$ and $7 \omega$ forcing components have roughly equal effect. Here numerous new instability tongues corresponding to modes with dominant frequencies $\omega / 2, \omega, 3 \omega / 2,2 \omega, \ldots$ are present due to the interaction of the two forcing components-it is not simply a superposition of the two previous cases. Although none of these new modes are parametrically forced (at leading order in $a$ ), they can interact with the two forced modes to influence the pattern selection process, particularly if they are weakly damped.

\section{B. Nonlinear selection}

As the forcing amplitude surpasses the minimum of the first instability tongue, a transition from a flat surface to a patterned state takes place. Since the infinitely extended system has planar Euclidean symmetry (which includes rotations), the flat state does not simply lose stability to a single eigenfunction, but to an entire circle of eigenfunctions of the form $\exp \{i \mathbf{k} \cdot \mathbf{r}\}$, where $\mathbf{r}=(x, y)$ and $\mathbf{k}$ is a wave vector of arbitrary orientation with magnitude $k_{c}$. In other words, the eigenfunctions are stripe patterns orientated in any direction in the plane. Any superposition from this infinite set of eigenfunctions is a solution of the linear problem. It is only at the nonlinear level that a finite set of these can be selected. Presupposing such a selection allows amplitude equations to be derived, although one is then limited to describing the competition between patterns associated with subsets of this selection. In the simplest possible case, where two eigenfunctions of the form $A_{1} \exp \left\{i k_{c} x\right\}$ and $A_{2} \exp \left\{i k_{c}(x \cos \theta+y \sin \theta)\right\}$ are considered, the amplitude equations take the form (see, e.g., [10]),

$$
\begin{aligned}
& \dot{A}_{1}=\lambda A_{1}+A_{1}\left(s\left|A_{1}\right|^{2}+b(\theta)\left|A_{2}\right|^{2}\right)+\cdots, \\
& \dot{A}_{2}=\lambda A_{2}+A_{2}\left(s\left|A_{2}\right|^{2}+b(\theta)\left|A_{1}\right|^{2}\right)+\cdots,
\end{aligned}
$$

where $\lambda$ is a parameter that measures the distance from onset, $s$ is the self-interaction coefficient, and $b(\theta)$ is the cross-coupling coefficient. Equations (5) contains two types of steady solutions: stripes $\left(A_{1}, A_{2}\right)=(A, 0)$ and rectangles $\left(A_{1}, A_{2}\right)=(A, A)$. The signs of $s$ and $s+b$ determine whether these two states bifurcate supercritically or subcritically, while the sign of $s-b$ determines their relative stability. If $s-b$ is positive then stripes are preferred to rectangles; vice versa if $s-b$ is negative. Such arguments can be extended to amplitude equations involving more modes to account for more complicated states, such as superlattice patterns [9].

If the excitation is single frequency, or effectively dominated by one component, then only simple patterns such as stripes, squares, and hexagons are observed in experiment, and it is straightforward to obtain the form of the appropriate amplitude equations from symmetry arguments. The influence of the additional modes that appear when multiple frequencies are important leads to more complex patterns that, in principle, demand more complicated equations. However, because the most important interactions are resonant triads, that is, interactions among three waves with wave vectors satisfying $\mathbf{k}_{1} \pm \mathbf{k}_{2}=\mathbf{k}_{3}$, the simple cubic order amplitude equations [Eqs. (5)] contains a surprising amount of general information about the pattern selection process. In particular, whenever the resonant triad involves two excited modes and a single damped mode (as is often the case), then its effect is manifest in the cubic cross-coupling coefficient $b(\theta)$, which can change dramatically as the interaction angle $\theta$ approaches the resonant value $[10,18]$.

Resonant triad interactions may involve damped modes with wave number $k_{d}<k_{c}$ or $k_{d}>k_{c}$. Due to the symmetry of $b(\theta)$ about $\theta=90^{\circ}$, however, it is convenient to restrict consideration to $\theta \in\left[0,90^{\circ}\right]$ and to divide resonant triads into "sum" and "difference" cases, accordingly; both of these are illustrated in Fig. 2. Note that the difference case describes triad interactions involving longer wavelength modes $\left(k_{d}<k_{c}\right)$ for $\theta<60^{\circ}$ and shorter wavelength modes $\left(k_{d}>k_{c}\right)$ for $\theta>60^{\circ}$. The sum case always describes triad interactions with shorter wavelength modes $\left(k_{d}>k_{c}\right)$. The positions of the instability tongues provide the values of $k_{c}$ and $k_{d}$ that can be used to calculate the resonance angle $\theta$. In the difference case of Fig. 2(a)

$$
\sin \frac{\theta}{2}=\frac{k_{d}}{2 k_{c}},
$$

and in the sum case of Fig. 2(b)

$$
\cos \frac{\theta}{2}=\frac{k_{d}}{2 k_{c}} \text {. }
$$
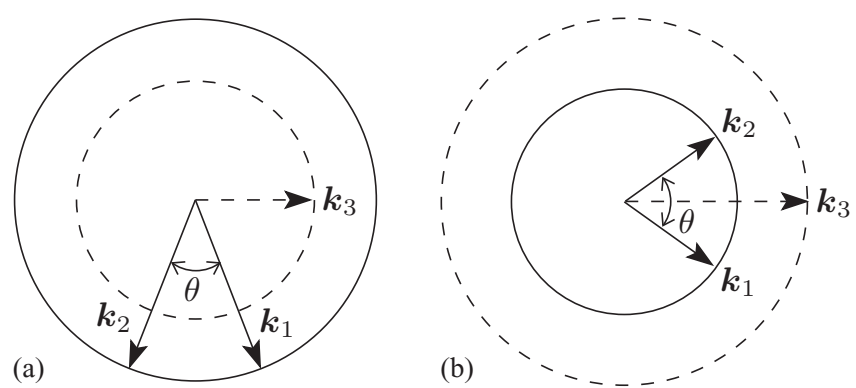

FIG. 2. Resonant triad interactions satisfying (a) $\boldsymbol{k}_{1}-\boldsymbol{k}_{2}=\boldsymbol{k}_{3}$ and (b) $\boldsymbol{k}_{1}+\boldsymbol{k}_{2}=\boldsymbol{k}_{3}$, with $\left|\boldsymbol{k}_{1}\right|=\left|\boldsymbol{k}_{2}\right|=k_{c}$ and $\left|\boldsymbol{k}_{3}\right|=k_{d}$. 
Note that, because of the discrete time translation symmetry, a damped mode can only interact with two critical modes if it is harmonic.

One can use a knowledge of $b(\theta)$ to discover the important triad resonances and their effect (enhancing or suppressing), then use this to infer the preferred pattern angles. For example, in [5], for one set of excitation parameters, superlattice patterns with two apparent length scales related by a factor $\sqrt{ } 7$ are reported. The spatial Fourier spectrum of these superlattice patterns reveals that they consist of modes from two hexagonal states rotated by approximately $22^{\circ}$ with respect to each other. The presence of this particular angle can be directly related to the fact that there is a peak in the $b(\theta)$ curve at approximately $22^{\circ}$. For another set of parameter values the same experiment yields quasipatterns. The spatial Fourier spectrum of these quasipatterns reveals two sets of hexagonal states rotated by approximately $30^{\circ}$ with respect to each other, a result of the peak in the $b(\theta)$ curve shifting upward to this value [14].

Given the attention that superlattice patterns and quasipatterns receive in experiments (likewise in the current paper), a brief discussion is warranted here. Superlattice patterns are so named because they exhibit a spatial periodicity that quasipatterns do not. This distinction, however, may not be particularly helpful as both kinds of patterns have much in common. For instance, both the superlattice patterns and the quasipatterns mentioned above can be thought of as (nonlinear) superpositions of hexagons rotated with respect to each other, and in each case their presence can be understood through the same kind of resonant triad interaction. In principle, patterns could be formed for any angle: Some angles would give spatially periodic patterns and some would not. For $\theta=30^{\circ}$ the superposition of two hexagonal patterns yields quasipatterns, but for an angle arbitrarily close to $\theta=30^{\circ}$, there is a superlattice pattern with a very long spatial periodicity that would be indistinguishable from the "true" quasipattern. Both superlattice patterns and quasipatterns have been studied by considering amplitude equations for a finite number of modes [18,19,21]. From a mathematical point of view, the issue of small divisors raises questions about the validity of studying quasipatterns via amplitude equations [20]; the reduction of a spatially periodic pattern to amplitude equations, in contrast, can be formally justified. Again, it might be argued that this distinction is largely academic because, for a superlattice pattern to be visually indistinguishable (over a large spatial domain) from a "true" quasipattern, the set of parameters over which the amplitude equations are formally justified would be vanishingly small. As shown in [19], in spite of the theoretical questions about its mathematical validity, an amplitude equation approach to quasipatterns remains useful. In light of these considerations, the term "superlattice" will be used more broadly in what follows than "quasipattern," but it should be understood that there are quasipatterns arbitrarily close to any given superlattice pattern, and vice versa.

It is also worth remarking here that the use of multifrequency forcing invalidates the intuitive argument that resonant triads involving damped modes should function as energy sinks and, therefore, that selected patterns should avoid them. Such reasoning can be found in [21], for example, where the effects of triad interactions on the competition among regular patterns (squares, hexagons, quasipatterns) is examined with the aid of a Lyapunov function in the low damping limit and with single-frequency forcing. Although this intuitive argument is compelling and likely correct for single-frequency forcing, it does not extend to the multifrequency forcing case, as seen below, where certain damped modes can actually promote the patterns they interact with $[11,18]$.

\section{RESONANCE EFFECTS AND THE LIMITATIONS OF LOW DAMPING THEORY}

In this section we investigate the effects of resonant triad interactions on pattern formation via the cubic cross-coupling coefficient $b(\theta)$, or the quadratic interaction coefficient $\alpha$ in the case of hexagons. In particular, by comparison with results from the NS equations, we aim to determine how well these effects are described by the ZV equations and by more recent symmetry-based theory $[11,12]$ as damping is increased. All calculations use $\rho=0.950 \mathrm{~g} / \mathrm{cm}^{3}$ and $\sigma=20.6 \mathrm{~g} / \mathrm{s}^{2}$, values appropriate for certain silicone oils and, in particular, those used by [5]. The values used for $\omega$ are also motivated by those used in [5].

\section{A. Resonant features with two-frequency excitation}

We first consider two-frequency forcing, focusing on the ratio $m: n=6: 7$ and the mixing angle $\chi=55^{\circ}$ used for the neutral stability calculation of Fig. 1(b). This choice of forcing frequencies has received much attention since superlattice patterns were discovered [5] with this ratio. The most interesting patterns appear when the $6 \omega$ component is dominant [i.e., when the dominant modes are harmonic (primary frequency $3 \omega$ )], and this will be assumed in what follows. The value of $55^{\circ}$ was chosen so that, for experimentally relevant parameters like those used in Fig. 1, the forcing of the odd component (the $7 \omega$ component) was as large as possible, to promote the resonant triad interaction with the damped $n-m$ mode, without being so large that it dominates. Decreasing $\chi$ reduces the overall height of the resonance peak in $b(\theta)$ while increasing $\chi$ soon renders the primary instability subharmonic rather than harmonic. In fact, for both the NS and $\mathrm{ZV}$ equations, this crossover to subharmonic primary instability already occurs with $\chi=55^{\circ}$ for small $\gamma$ (less than about 0.05 ). Nonetheless, for simplicity, we opted to retain the same value of $\chi$ throughout this and subsequent sections. This does not generate any difficulties, or have any bearing on the comparisons presented here, because quadratic interaction with the subharmonic modes is forbidden by symmetry and their onset does not cause singularities in the coefficients of Eqs. (5) describing the harmonic modes.

The symmetry arguments of $[11,12,22]$ predict which resonant triad interactions should be important in the limit of weak damping. In particular, for 6:7 excitation one expects three different resonance values of $\theta$ to feature prominently in the graph of $b(\theta)$ :

(i) At $\theta=60^{\circ}$ three modes on the critical circle form a resonant triad. This resonance is associated with hexagons, and with a singularity in $b(\theta)$ since only two of the three critical modes are considered in Eqs. (5).

(ii) At $\theta \approx 22^{\circ}$ a resonant triad is formed between two critical modes and the " $n-m$ " (i.e., $\omega)$ mode $\left(k_{d} \approx 0.35\right.$ ). 
(a)

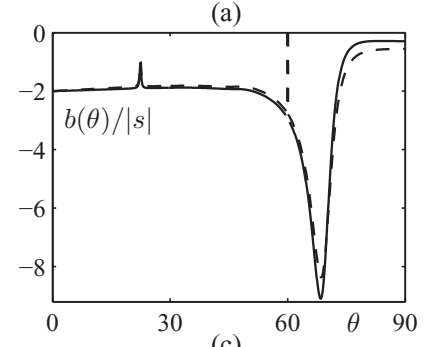

(c)
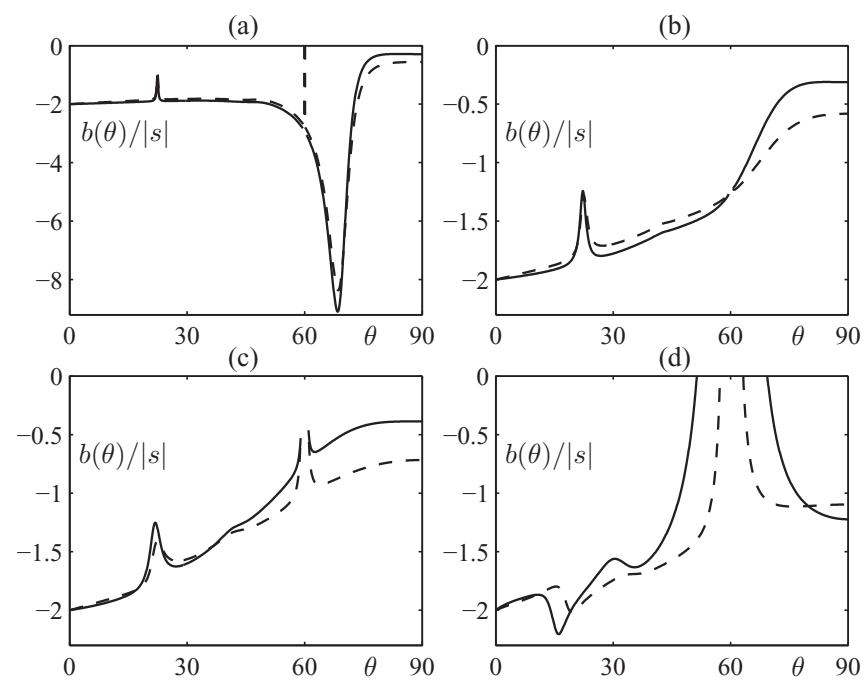

FIG. 3. The cross-coupling coefficient $b(\theta) /|s|$ for $m: n=6: 7$ excitation, $\phi_{m}=\phi_{n}=0, \chi=55^{\circ}, G_{0}=0.0396, \Gamma_{0}=0.2104$. The solid curves are computed from the NS equations and the dashed curves from the $\mathrm{ZV}$ model. (a) $\gamma=0.01$, (b) $\gamma=0.05$, (c) $\gamma=0.1$, (d) $\gamma=0.5$.

This interaction favors related patterns, that is, leads to an increase in $b(\theta)$, and scales as $\left|f_{n}\right|^{2} / \gamma[22]$.

(iii) At $\theta \approx 70^{\circ}$ a resonant triad is formed between two critical modes and the " $m$ " (i.e., $6 \omega)$ mode $\left(k_{d} \approx 1.6\right)$. This resonance causes a dip in $b(\theta)$ and discourages related patterns. Its magnitude is predicted to scale as $1 / \gamma$ [22].

Figure 3 shows $b(\theta) /|s|$, computed both from the NS and $\mathrm{ZV}$ equations, for four different values of the dimensionless viscosity $\gamma$. In Fig. 3 one can see all three features described above. At low viscosity (a), there is a (narrow) peak at approximately $22^{\circ}$ and a large dip at approximately $70^{\circ}$, as well as a singularity at $60^{\circ}$ (an interval near $60^{\circ}$ is removed in subsequent plots). The NS and ZV equations agree in this regime, as expected. At the moderate viscosity of (c), which is representative of the experiments of [5], the $\mathrm{ZV}$ equations begin to underestimate $b(\theta)$, although still correctly capturing its basic behavior including the peak near $22^{\circ}$. Note that the resonance (dip) near $70^{\circ}$ is no longer important. Upon further increase of the viscosity (d) the former peak due to triad resonance with the $n-m$ mode broadens and transforms into something more like a dip followed by a smaller maximum, signaling the failure of the low damping theory.

The height and width of the resonant feature near $22^{\circ}$ and that near $70^{\circ}$ were calculated by fitting a surrounding interval to the function

$$
b(\theta)=c+d \theta+e \theta^{2}+\frac{h}{1+4\left[\left(\theta-\theta_{d}\right) / w\right]^{2}} .
$$

The fit parameter $h$ defines the height, $w$ the width, and $\theta_{d}$ the resonance location. Results for the width and height for the features at approximately $22^{\circ}$ and $70^{\circ}$ are shown in Figs. 4 and 5 , respectively, as a function of $\gamma$ for both the NS and ZV equations. The results in Fig. 4 demonstrate that the predicted scaling of the height of the resonant feature holds well for $\gamma \lesssim$ 0.01 , remains in reasonable qualitative agreement until $\gamma \approx$ (a)

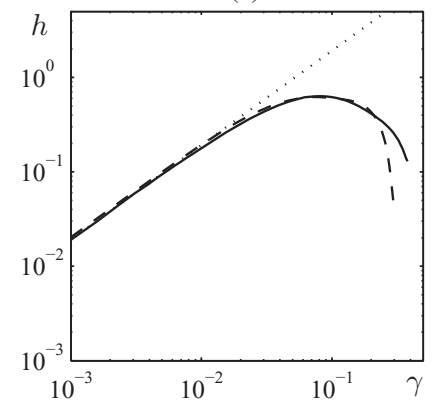

(b)

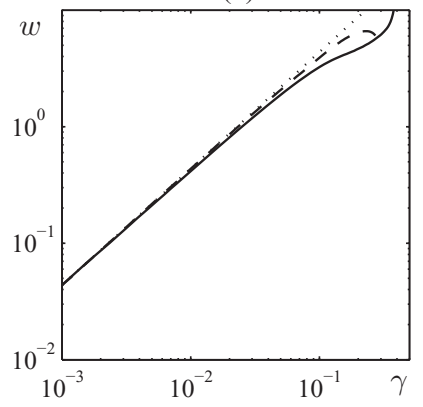

FIG. 4. Dependence on $\gamma$ of the resonant feature near $22^{\circ}$, calculated from the NS (solid curves) and ZV (dashed curves) equations: (a) height $h$, (b) width $w$. The dotted lines show the linear scaling predicted by theory. Remaining parameters are as in Fig. 3.

0.1, but fails thereafter: Note, for example, how in Fig. 3(d) the former peak is more of a dip by $\gamma=0.5$.

In Fig. 5(a) we see that the peak at $70^{\circ}$ diminishes with increasing damping, as predicted. By $\gamma=0.04$ the peak has essentially disappeared. It is apparent that this is a low damping resonance feature that is captured well, both qualitatively and quantitatively, by the ZV equation and by the theoretical scaling predictions.

\section{B. Estimating resonance angles}

The height of the peak in $b(\theta)$ determines the extent to which a particular resonant triad influences pattern selection, but the position of the peak determines which pattern(s) it influences: The fact that superlattice patterns with two apparent length scales in the ratio of $\sqrt{ } 7$ were found in [5] can be related directly to the fact that there is a peak in the $b(\theta)$ curve at $22^{\circ}$ for the relevant parameter values. The position of the peak depends on the damping, which is apparent from the solid curves in Figs. 6(a) and 6(b), as well as the other parameters in the model. At issue in this section is how, for given fluid parameters, one can best figure out where the peak will be and hence what patterns to expect.

The easiest way to estimate the resonance angle is to appeal to the inviscid problem and calculate $k_{d}$ from the dispersion (a)

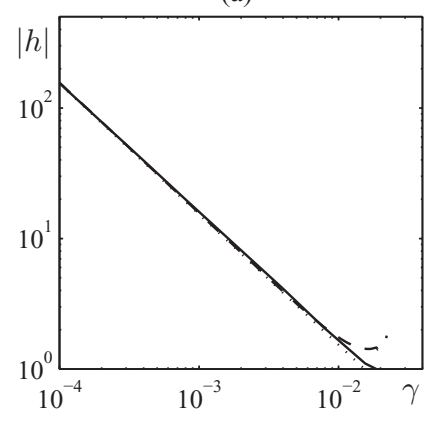

(b)

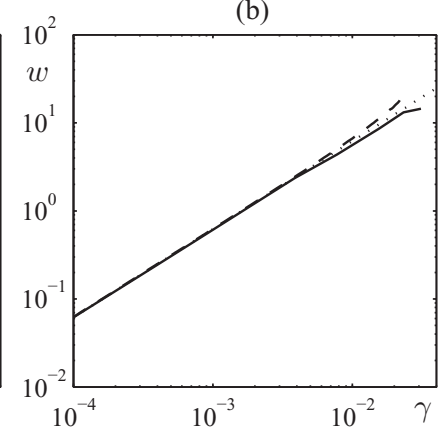

FIG. 5. (a) Height $h$ and (b) width $w$ of resonant feature near $70^{\circ}$ as a function of $\gamma$. By $\gamma \sim 0.02$ the peak is very broad and the fitting function (8) no longer works well. The solid (dashed) curves show the results for the NS (ZV) equation while the dotted line shows the theoretical prediction. Remaining parameters are as in Fig. 3. 
(a)

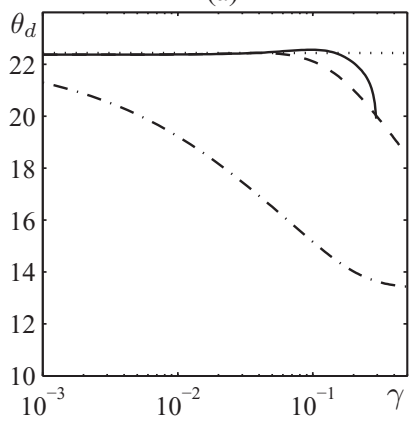

FIG. 6. The resonant angle $\theta_{d}$ calculated (parameters as in Fig. 3 from fitting $b(\theta)$ with Eq. (8) (solid curve) compared with predictions based on the following: the ratio of the wave number of the critical model and the wave number of the harmonic mode that is closest to critical (dashed curve); the location of the minimum of the $\omega$ instability tongue (dot-dashed curve); the value associated with the inviscid limit (dotted line). (a) ZV; (b) NS.

relation (1), which gives a value that is independent of $\gamma$. The estimate from Eq. (6) of $\theta_{d}=22.43$ is indicated by a dotted line in Fig. 6 and performs surprisingly well for $\gamma \leqslant 0.1$.

Another obvious scheme for predicting the angle $\theta_{d}$ relies on locating the minimum of the relevant linear stability tongues. For example, the two modes involved in the resonant triad interaction that produces the peak near $22^{\circ}$ in the $b(\theta)$ curve onset with $k_{c}=0.9719$ and $k_{d}=0.2516$ when $\gamma=0.1$ [see Fig. 1(b)]. Using Eq. (6), this leads to an estimate for the resonant angle of $\theta_{d} \approx 15^{\circ}$. As indicated by the dot-dashed curves in Fig. 6, this method works well only for very small values of $\gamma$ and provides a poor estimate with moderate values like that used to produce Fig. 1. This surprising failure shows that the location of the minimum of the instability tongue for the resonant mode is not always relevant-this tongue may sit at forcing values much greater than $a_{c}$, where additional detuning is significant.

A third method is based on finding the wave number of the damped mode with Floquet multiplier closest to critical at $a=a_{c}$. Since the forcing is set to the critical value, this mode ought to be more relevant than that defined by the (higher) minimum of the instability tongue. With the parameter values of Fig. 1(b), the real parts of the two most dangerous Floquet

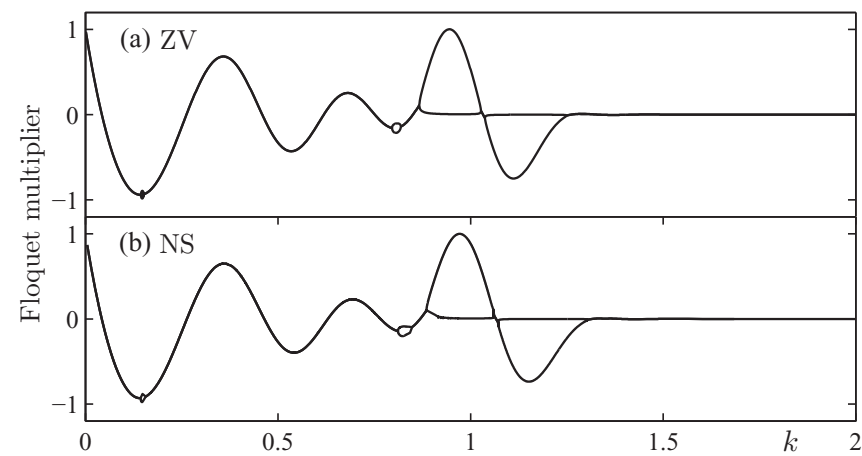

FIG. 7. The real part of the two Floquet multipliers closest to critical at $a=a_{c}, \gamma=0.1$. (a) For the ZV equation, $a_{c}=7.9504$. (b) For the NS equation, $a_{c}=5.7846$. Remaining parameters are as in Fig. 3. multipliers at $a=a_{c}$ are as shown in Fig. 7, which compares computations from the ZV equations (a) and the NS equations (b). These Floquet multipliers rotate around the complex plane as $k$ is increased, reflecting the increase in "natural" frequency, and spiral toward the origin, reflecting the increase in effective damping. Successive crossings of the real axis can be identified with damped subharmonic or harmonic resonances; these are evident in Fig. 7 by successive approaches to -1 and 1 , respectively. Most of the time, the Floquet multipliers are complex conjugate pairs, which explains why only one curve can be seen for most values of $k$ in Fig. 7. However, near the maxima and minima (i.e., near resonance values) the most dangerous Floquet multipliers split into a pair of real eigenvalues, giving the appearance of bubbles in Fig. 7. The "bubble" is very large near the most critical mode $(k \simeq 1)$, but is hardly visible on the scale of the plot for the first maximum, near $k=0.35$. For the NS equation, the critical mode (Floquet multiplier equal to 1 ) occurs at $k_{c}=0.9719$. The value of the Floquet multiplier for the weakly damped mode at $k_{d}=0.3689$ is 0.64 , which leads to a prediction of $\theta_{d}=21.88^{\circ}$. In Fig. 6 the value of $\theta_{d}$ calculated in this manner from the Floquet multipliers is shown as a dashed curve.

In the case of the ZV equations, one final method suggests itself. This approach is based on the fact that the resonant interaction can only occur with real Floquet multipliers, not complex ones, and bounds on the region containing real Floquet multipliers (the bubbles in Fig. 7) can be calculated as follows.

For the $\mathrm{ZV}$ equation the linear stability problem reduces to a Mathieu equation

$$
\ddot{p}_{k}+2 \gamma k^{2} \dot{p}_{k}+\left(\gamma^{2} k^{4}+G_{0} k+\Gamma_{0} k^{3}\right) p_{k}=k f(t) p_{k} .
$$

Since $f(t)$ is periodic, Eq. (9) has solutions of the form

$$
p_{k}(t)=\mathrm{e}^{\lambda t} \sum_{r} b_{r} \mathrm{e}^{i r \omega t}+\text { c.c. },
$$

where $\lambda$ is the Floquet exponent and c.c. denotes complex conjugate. Substitution of Eq. (10) into Eq. (9) results in the generalized eigenvalue problem,

$\left[(\lambda+i r \omega)^{2}+2(\lambda+i r \omega) \gamma k^{2}+\gamma^{2} k^{4}+\Omega^{2}\right] b_{r}=k B_{r}$,

where $\Omega^{2}=G_{0} k+\Gamma_{0} k^{3}$ and $B_{r}$ is the $r$ th Fourier coefficient of $\left\{f(t) \exp (-\lambda t) p_{k}(t)\right\}$. In order to find harmonic marginal stability points $\lambda$ is set to 0 , and Eq. (11) reduces to

$$
\left[(i r \omega)^{2}+2(i r \omega) \gamma k^{2}+\gamma^{2} k^{4}+\Omega^{2}\right] b_{r}=k B_{r} .
$$

(This is not the best way to solve the Mathieu instability tongue problem - a computationally efficient method is given in [23]).

Here we are interested in finding an estimate for the position of the bubbles of real Floquet multipliers, specifically those that occur on the harmonic tongues as seen in Fig. 7. Using Floquet theory [24], the product of the Floquet multipliers $\mu_{i}, i=1,2$ for Eq. (9) is given by

$$
\mu_{1} \mu_{2}=\exp \left(-2 \gamma k^{2} T\right)
$$

where $T$ is the period of $f(t)$. The transition from real to complex Floquet multipliers occurs when

$$
\mu_{1}=\mu_{2}=\exp \left(-\gamma k^{2} T\right) .
$$


The Floquet multipliers are related to the Floquet exponents by $\mu_{i}=\mathrm{e}^{\lambda_{i} T}$. Hence, for harmonic tongues, at the transition from real to complex Floquet multipliers, both Floquet exponents are equal to $-\gamma k^{2}$. Using $\lambda=-\gamma k^{2}$ Eq. (11) reduces to

$$
\left[(i r \omega)^{2}+\Omega^{2}\right] b_{r}=k B_{r} .
$$

This is exactly Eq. (12) in the case when $\gamma=0$. Consequently, a single calculation of the Mathieu instability tongues for the inviscid case $\gamma=0$ also gives the position of the transition points for the harmonic tongues in the damped problem. Although the bubble is not exactly symmetric, taking the midpoint between the two transition points at $a=a_{c}$ provides a decent estimate of the wave number $k$ where the real Floquet multiplier is maximal. This method can be extended to enable the calculation of the position of the bubbles for the subharmonic case, where subharmonic tongues can be computed by setting $\lambda=\frac{i \omega}{2}$ in Eq. (11) and the corresponding condition for transition from real to complex Floquet multipliers is $\mu_{1}=\mu_{2}=-\exp \left(-\gamma k^{2} T\right)$.

In Fig. 8(a) the inviscid instability tongue corresponding to the first damped harmonic mode is shown in the $(k, a)$ plane. This instability tongue can then be mapped into the $\left(\theta_{d}, \gamma\right)$ plane in order to give a prediction of the resonant angle, shown in Fig. 8(b), as follows. For each value of $\gamma$ the value of $a_{c}$ is calculated. For this value of $a_{c}$, Fig. 8(a) gives two values for $k$, one on each side of the tongue. These values of $k$ then correspond to two different values of $\theta_{d}$, as given by Eq. (6).

The same argument cannot be used for the NS equation: In [25] it is shown that the viscous NS equations lead to a Mathieu equation with nonlocal terms for which there is no result analogous to Eq. (13). However, it is interesting to (a)

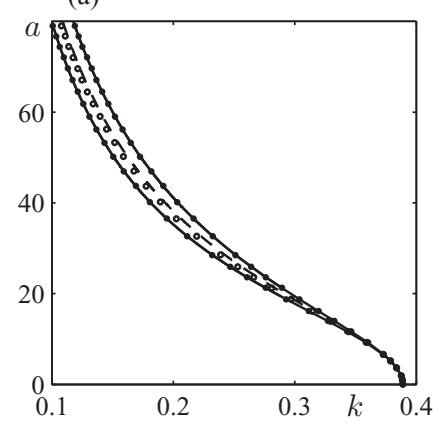

(b)

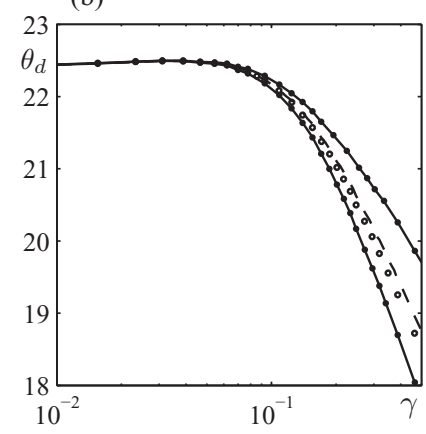

FIG. 8. (a) The points where the Floquet multipliers change from complex to real for the $\omega$ instability tongue with $m: n=6: 7$ and other parameters as in Fig. 3. The solid curves show the edges of this tongue as calculated from Eq. (14) and the dashed curve marks the center of this tongue. The dots, which lie on top of the solid curves, and the open circles in between are from an explicit calculation of the Floquet multipliers for the damped $\mathrm{ZV}$ equation, similar to those used to produce Fig. 7-the dots show where the Floquet multipliers change from real to complex, and the open circles show where the Floquet multiplier reaches its maximum value. (b) Mapping of resonance tongue onto the $\left(\gamma, \theta_{d}\right)$ plane to give predictions for $\theta_{d}$. The solid curves (and dots) give bounds on the possible values for $\theta_{d}$ and correspond to the boundaries of the tongue in (a). The dashed curve is the image of the center of this tongue. The open circles give the estimate of $\theta_{d}$ as calculated from the Floquet multipliers.

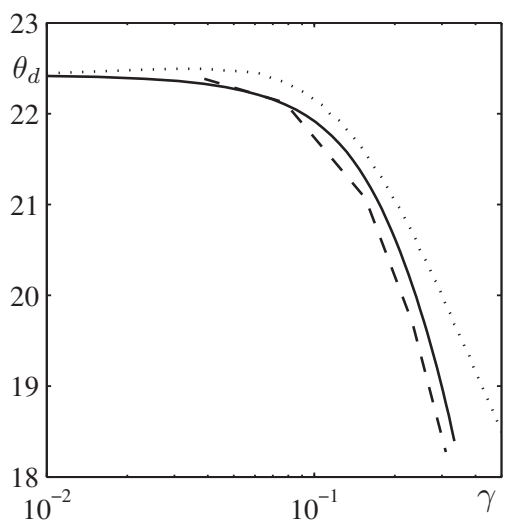

FIG. 9. NS prediction of $\theta_{d}$ using the Mathieu tongue mapping technique (dotted curve) compared with the $b(\theta)$ results (solid curve) and the results from the Floquet multipliers for the NS equation (dashed curve). Other parameters are as in Fig. 3.

note that applying the same Mathieu equation technique via Eq. (14) still gives a good estimate for $\theta_{d}$ in the NS case, even at high values of $\gamma$, as shown in Fig. 9. This estimate is obtained with the same mapping technique described above in connection with Fig. 8(b), using the tongue from the inviscid approximation and the $\gamma$ versus $a_{c}$ relationship for the Navier-Stokes equation.

This Mathieu tongue mapping technique gives a good estimate of the resonant angle. It is also an easy method to apply, requiring only one computation of $a_{c}$ versus $\gamma$ and one computation of the Mathieu tongue for the inviscid approximation. It is significantly easier than calculating $b(\theta)$ or calculating the Floquet multipliers directly.

\section{Quadratic coefficient for hexagons}

A great deal can be learned about the relative likelihood of observing different patterns from the shape of the $b(\theta)$ curve, which captures the influence of damped resonant modes via triad interactions. However, Eqs. (5) is limited by the fact that only two critical modes are assumed to interact. This assumption fails for hexagons, as revealed by the singularity at $60^{\circ}$ in Fig. 3. To treat hexagons, where a resonant triad is formed among three critical modes, requires a different approach. By associating $A_{1}, A_{2}$, and $A_{3}$ with the three critical eigenmodes with eigenvectors orientated at $120^{\circ}$ with respect to each other, one arrives at the amplitude equation

$$
\begin{aligned}
\dot{A}_{1}= & \mu A_{1}+\alpha \bar{A}_{2} \bar{A}_{3}+A_{1}\left(b_{1}\left|A_{1}\right|^{2}+b_{2}\left|A_{2}\right|^{2}\right. \\
& \left.+b_{2}\left|A_{3}\right|^{2}\right)+\cdots
\end{aligned}
$$

Evolution equations for $A_{2}$ and $A_{3}$ are obtained by cyclic permutation of indices. Of particular importance in this equation is the magnitude of the quadratic coefficient $\alpha$, which reflects the strength of the resonant triad interaction (unless it is forbidden by other symmetry considerations). The value of $\alpha$, and of the other coefficients, depends on the fluid properties and on the form of the forcing, including the relative phase of its different components. The dependence of pattern selection on the forcing phases has been seen experimentally in [4,5]. Using symmetry arguments, [11] derived the expected phase 
dependence for the quadratic (and cubic) coefficients. Namely, if the excitation is given by Eq. (3) with $m$ even, then

$$
\alpha= \begin{cases}c_{1}\left|f_{n}\right|^{m / 2} \cos \Phi, & n=1 \\ c_{2}\left|f_{n}\right|^{m / 2}\left|f_{m}\right|^{(n-3) / 2} \sin \Phi, & n>1,\end{cases}
$$

where $c_{1}$ and $c_{2}$ are real $O(1)$ coefficients and

$$
\Phi=\frac{\pi}{4}-\frac{1}{2}\left(n \phi_{m}-m \phi_{n}\right) .
$$

In [11] [where an oppositely signed forcing term means that $\cos \Phi$ and $\sin \Phi$ switch places in Eq. (16)] these results were validated by comparing with results from the $\mathrm{ZV}$ equation for specific choices of $m: n$. Below we compare with results from the NS equations and the $\mathrm{ZV}$ equations for the same choices of $m: n$, namely $2: 3$ and $2: 1$. If $m: n=2: 1, \phi_{2}=0$, and $\phi_{1}=\phi$ then the scaling of the quadratic coefficient $\alpha$ reduces to

$$
\alpha=c_{1}\left|f_{1}\right| \cos \Phi,
$$

where $\Phi=\frac{\pi}{4}+\phi$, while if $m: n=2: 3, \phi_{2}=0$, and $\phi_{3}=\phi$, then this scaling is

$$
\alpha=c_{2}\left|f_{3}\right| \sin \Phi \text {. }
$$

In Fig. 10 the value of $\alpha$ as a function of $\Phi$ is shown for several different values of $\gamma$. It can be seen that for low values of $\gamma$ (the solid curves), the dependence is sinusoidal and reaches extrema in the positions predicted by [11], namely at $\Phi=0^{\circ}, \pm 180^{\circ}$ in the case of $2: 1$ excitation and at $\Phi= \pm 90^{\circ}$ in the case of $2: 3$ excitation. However, as $\gamma$ is increased, the amplitude of $\alpha$, the shape of the curve, and especially the

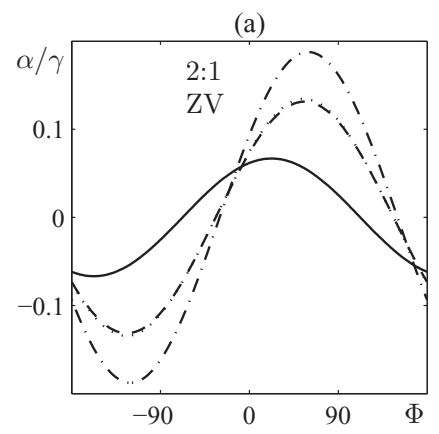

(c)
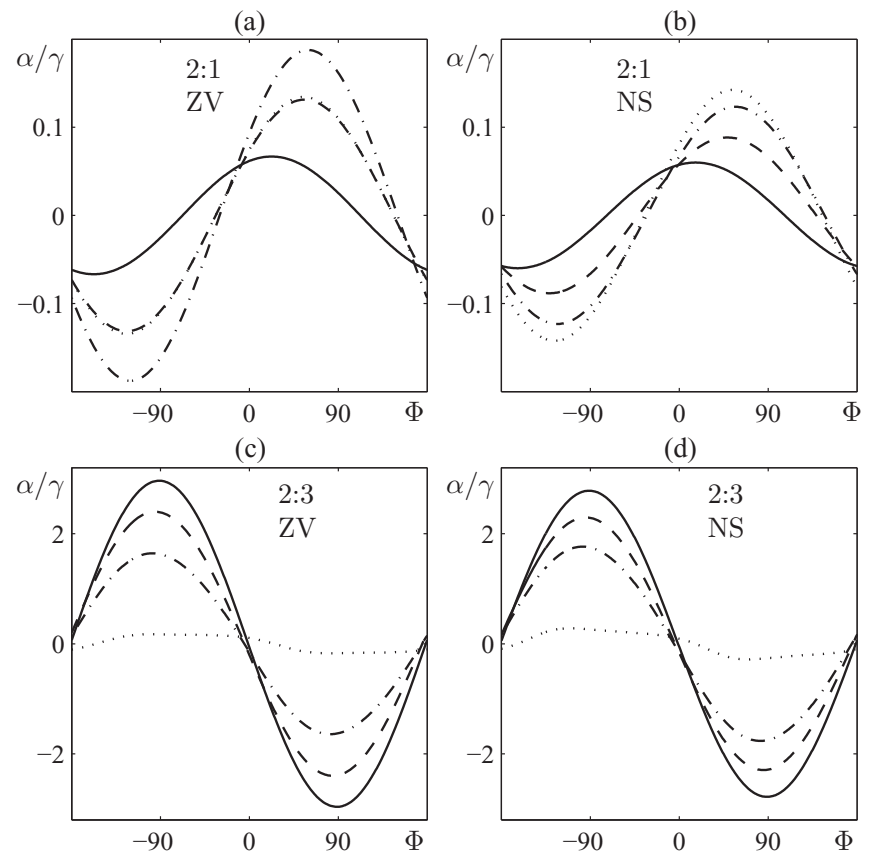

(d)

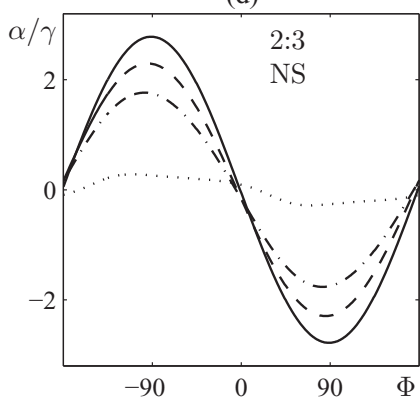

FIG. 10. Quadratic coefficient $\alpha$ as a function of phase $\Phi$ with $\omega=20 \pi, \rho=0.95 \mathrm{~g} / \mathrm{cm}^{3}, g=980 \mathrm{~cm} / \mathrm{s}^{2}, \sigma=20.6 \mathrm{dyn} / \mathrm{cm}$. (a) and (b) are calculated with $m: n=2: 1$ and $\chi=5^{\circ}$ using ZV and NS equations, respectively. (c) and (d) are calculated with $m: n=2: 3$ and $\chi=55^{\circ}$ using ZV and NS equations, respectively. The damping values used are as follows: $\gamma=0.01$ (solid curve), $\gamma=0.05$ (dashed curve), $\gamma=0.1$ (dot-dashed curve), $\gamma=0.5$ (dotted curve).
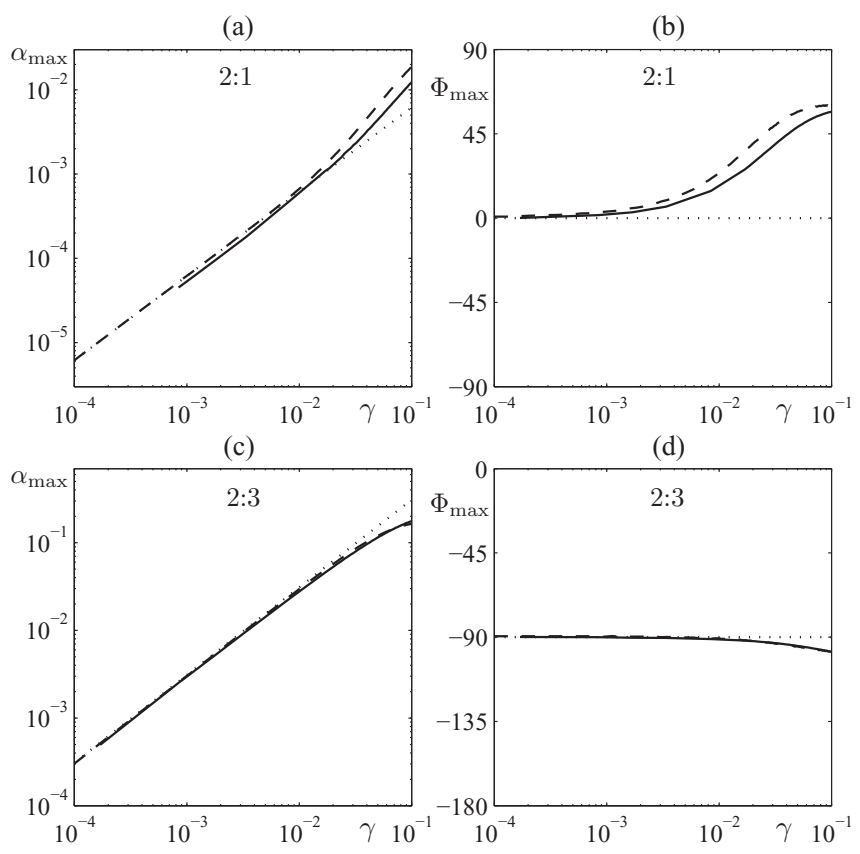

FIG. 11. Maximum of quadratic coefficient $\alpha$ as a function $\gamma$. The parameters are as in Fig. 10 with (a) and (b) calculated using $m: n=2: 1$ and (c) and (d) using $m: n=2: 3$. (a) and (c) Shows the maximum value of the quadratic coefficient, while (b) and (d) show the angle for which this maximum value occurs. The solid (dashed) curves are calculated from the NS (ZV) equations, while the dotted curve shows the theoretical prediction valid for small $\gamma$.

position of the extrema all change. Theory predicts that the amplitude depends linearly on $f_{n}$ and, since $f_{n} \propto \gamma$ for small $\gamma$, that the amplitude depends linearly on $\gamma$.

The amplitude of $\alpha$ and the position of its extrema are shown as functions of $\gamma$ in Fig. 11. While the amplitude $|\alpha|$ agrees well with the theoretical prediction for $\gamma$ less than 0.1 , the position of the extrema depends strongly on $\gamma$ beyond $\gamma \sim 0.01$, especially with $m: n=2: 1$. This dependence is not predicted in [11] since it results from the influence of higher order (in $\gamma$ ) terms, and probably explains some of the discrepancy between the theoretical results in [11] and certain experiments $[4,5]$ carried out at moderate values of $\gamma$.

We note that we have compared calculations of $\alpha$ from the NS and ZV equations for a number of other frequency combinations $m: n$ including 2:5, 2:7, 2:9, 4:1, 4:3, 4:5, 4:7, 6:1, $6: 5,6: 7,8: 3$, and 8:5. In all cases there was good agreement. The low viscosity dependence of $\alpha$ on $\gamma$ and $\Phi$ was also compared (using the ZV computations) with the theoretical predictions of Eq. (16) and found to be in agreement, with the exception of 4:3 excitation. In this particular case, although the NS and ZV equations agree with each other, the leading order term behind Eq. (16) appears to be absent. The theoretically predicted $\gamma^{2}$ scaling with damping is not observed (a higher order $\gamma^{3}$ dependence is found instead) and the maximum of $|\alpha|$ does not occur at $90^{\circ}$ (a maximum near $0^{\circ}$ is seen instead). It is tempting to speculate that the absence of the predicted term in this case is due to a hidden symmetry of some kind, but it may simply be that the predicted term is very small, for whatever reason, and would dominate at still lower values of $\gamma$ (where 
$\alpha$ becomes extremely small and numerical errors disrupt the calculation).

\section{Resonance features for three-frequency excitation}

Since the nature of the $b(\theta)$ curve reflects the relative preference of the system for different patterns, one way to control which patterns are selected is to control the resonances that underly the features of this curve. The easiest way to do that is by manipulating the form of the forcing function [12]. For example, if a third frequency is added to the excitation of Eq. (3) by taking

$$
\begin{aligned}
f(t)= & f_{m} \cos \left(m \omega t+\phi_{m}\right)+f_{n} \cos \left(n \omega t+\phi_{n}\right) \\
& +f_{p} \cos \left(p \omega t+\phi_{p}\right),
\end{aligned}
$$

with $m: n: p=6: 7: 2$, then the added $2 \omega$ component, which parametrically excites the damped $\omega$ mode, can be used to enhance its resonance effect by driving it closer to onset, provided an appropriate phase is used. As the $\omega$ mode becomes less and less damped, there is an increase in the cross-coupling coefficient at approximately $22^{\circ}$, as discussed in Sec. III A. This effect can be seen in Fig. 12, where the $b(\theta)$ curve for different values of $f_{2}$ are shown: Increasing $f_{2}$ does indeed increase the height of the feature at $\theta=\theta_{d}$, at least for low and moderate values of $\gamma$ [subplots (a)-(f)]. If $f_{2}$ is made large enough, the $\omega$ mode becomes bi-critical with the $3 \omega$ mode and a singularity develops in $b\left(\theta_{d}\right)$. In the case of Fig. 12 with $\gamma=0.01$, this happens at an amplitude $f_{2} \simeq 0.08$, so only curves with $f_{2}=0,0.04$ and 0.06 are shown. In the final case shown in Figs. 12(g) and 12(h), the viscosity parameter $\gamma$ is set to 0.5 , a value for which we have already seen in Fig. 3 that the resonant peak more resembles a dip. Including the forcing $f_{2}$ can accentuate this feature, but since a dip in the $b(\theta)$ curve is suppressing for related patterns rather than enhancing, adding the third frequency component would not facilitate the appearance of superlattice patterns for these high viscosities.

Not only does the amplitude of the additional third frequency in the excitation affect the resonant feature, so does its phase. This can be seen in Figs. 13(a) and 13(c) where $b(\theta)$ is obtained for $f_{2}=0.06$ and $\Phi= \pm 90$, and compared with the corresponding (dashed) curve for $f_{2}=0$ and $\Phi=0$. In Figs. 13(b) and 13(d) the dependence of $b\left(\theta_{d}\right)$ on $\Phi$ is shown over the full range. These results demonstrate that, although for most phases the inclusion of the third frequency component enhances the resonance feature, parametrically forcing the resonant mode does not always magnify its effect; in this case, for $\Phi$ between about $40^{\circ}$ and $135^{\circ}$, resonance suppression is observed.

According to [12], the height and width of the resonant peak at $b\left(\theta_{d}\right)$ with $m: n: p=6: 7: 2$ and $f_{2}$ fixed should scale with $\gamma$ in the low viscosity limit (just as with $f_{2}=0$ ). In addition, the height of the peak should be maximized at a phase $\Phi=$ $\phi_{2}+2 \phi_{6}-2 \phi_{7}=-90^{\circ}$; this differs in sign from [12] due to the different sign convention for the applied forcing used here. In order to examine the range of validity of these predictions, $f_{2}$ was fixed at 0.05 and the height, width, and position of the resonant feature were calculated; see Fig. 14. A picture similar to that seen for the 6:7 case emerges, namely that there is excellent agreement with theory at low viscosity $(\gamma<0.01)$,
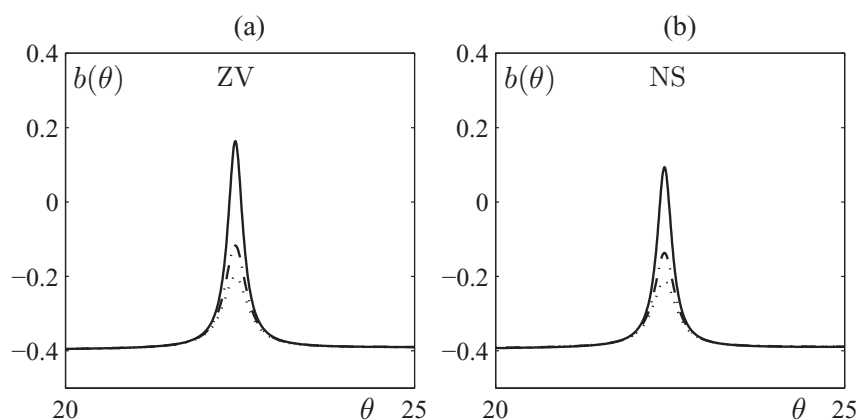

(c)
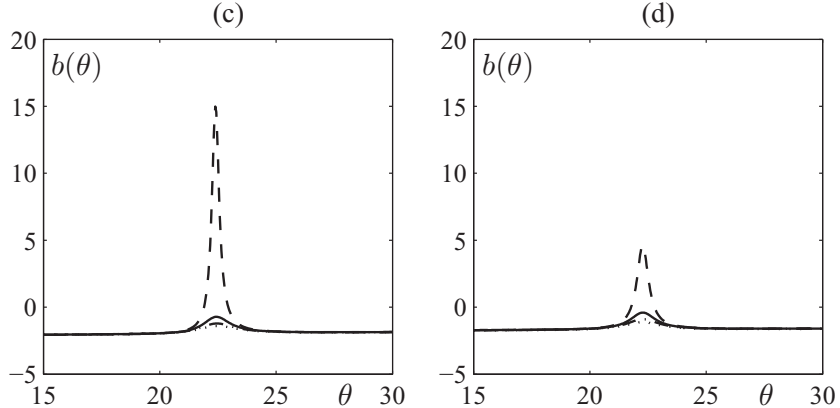

(e)
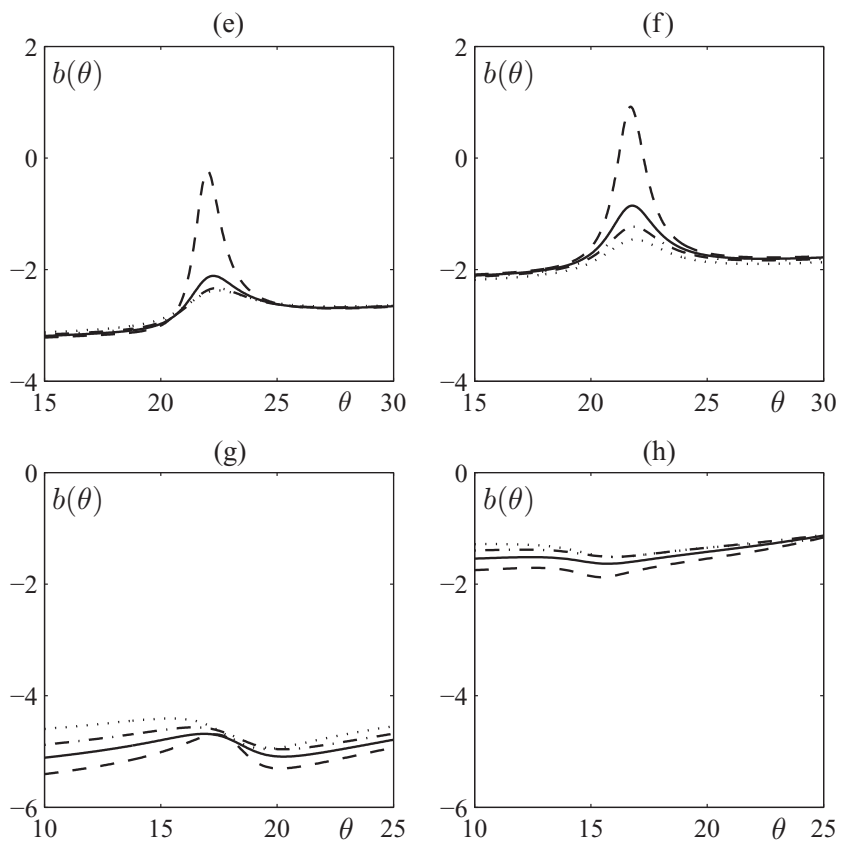

FIG. 12. $b(\theta)$ curves showing impact of $f_{2}$ with the forcing $\left(f_{6}, f_{7}, f_{2}\right)=a_{c} G_{0}\left(\cos 55^{\circ}, \sin 55^{\circ}, f_{2}\right)$ at various damping values: $\gamma=0.01$ for (a) and (b), $\gamma=0.05$ for (c) and (d), $\gamma=0.1$ for (e) and (f), $\gamma=0.5$ for (g) and (h). The values of $f_{2}$ used are as follows: $f_{2}=0$ (dotted curve), $f_{2}=0.04$ (dot-dashed curve), $f_{2}=0.06$ (solid curve), $f_{2}=0.08$ (dashed curve). The phases are equal, $\phi_{6}=\phi_{7}=\phi_{2}=0$, and the remaining parameters are as in Fig. 3. The ZV equations are used for (a), (c), (e), and (g) and the NS equations for (b), (d), (f), and (h).

qualitative agreement for moderate viscosity $(\gamma<0.1)$, but that at high viscosity the theory fails and the resonant feature is washed out. In fact, the maximum height of the resonant feature occurs for a $\gamma$ value near 0.1 .

In Fig. 15(a) the value of $\theta_{d}$ (calculated with null phases) is shown, while in Fig. 15(b) the value of $\Phi$ that maximizes $b\left(\theta_{d}\right)$ 

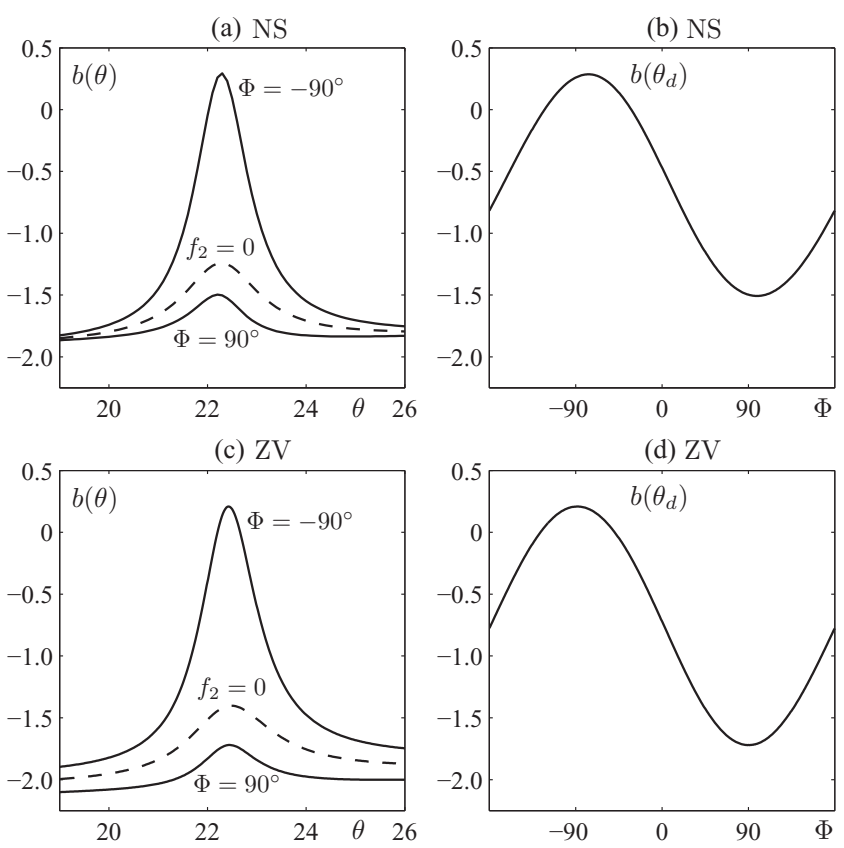

FIG. 13. Calculations from NS (a) and (b), and ZV (c) and (d), equations showing the effect of varying the phase $\Phi=\phi_{2}+$ $2 \phi_{6}-2 \phi_{7}$. At the predicted maximum, $\Phi=-90^{\circ}$, the resonance is much larger than at the predicted minimum, $\Phi=90^{\circ}$, where is it is actually reduced with respect to the unforced $\left(f_{2}=0\right)$ case shown as a dashed curve [12]. The expected sinusoidal behavior of $b\left(\theta_{d}\right)$ is evident in (b) and (d), which agree well with each other. The parameters used (except if $\left.f_{2}=0\right)$ are as follows: $\left(f_{6}, f_{7}, f_{2}\right)=$ $a_{c} G_{0}\left(\cos 55^{\circ}, \sin 55^{\circ}, 0.06\right), \phi_{6}=\phi_{7}=0, \phi_{2}=\Phi, \gamma=0.05, G_{0}=$ $0.0396, \Gamma_{0}=0.2104$.

is compared with the prediction of $-90^{\circ}$. Even at moderate viscosities, $\Phi_{\max }$ deviates substantially from this theoretical prediction and so, in this respect, behaves similarly to the quadratic (hexagon) coefficient $\alpha$ shown in Fig. 11. With increasing $\gamma$ the phase dependence deviates sooner from the small $\gamma$ theoretical prediction than do the remaining scaling laws. (a)

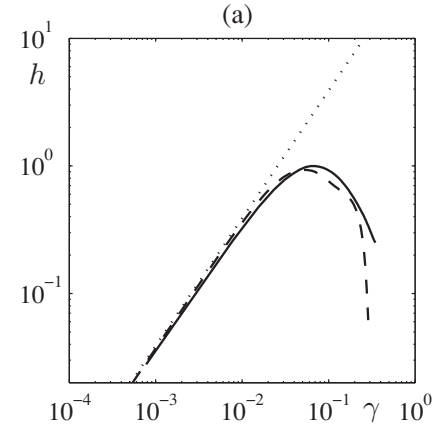

(b)

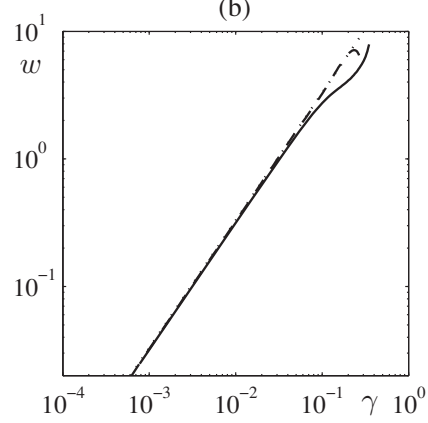

FIG. 14. Dependence on $\gamma$ of the resonant feature near $22^{\circ}$ with fixed forcing ratios $\left(f_{6}, f_{7}, f_{2}\right)=a_{c} G_{0}\left(\cos 55^{\circ}, \sin 55^{\circ}, 0.05\right)$, calculated from the NS (solid curves) and ZV (dashed curves) equations: (a) height $h$, (b) width $w$. The dotted lines show the linear scaling predicted by theory. The phases are equal, $\phi_{6}=\phi_{7}=\phi_{2}=0$, with remaining parameters as in Fig. 3.
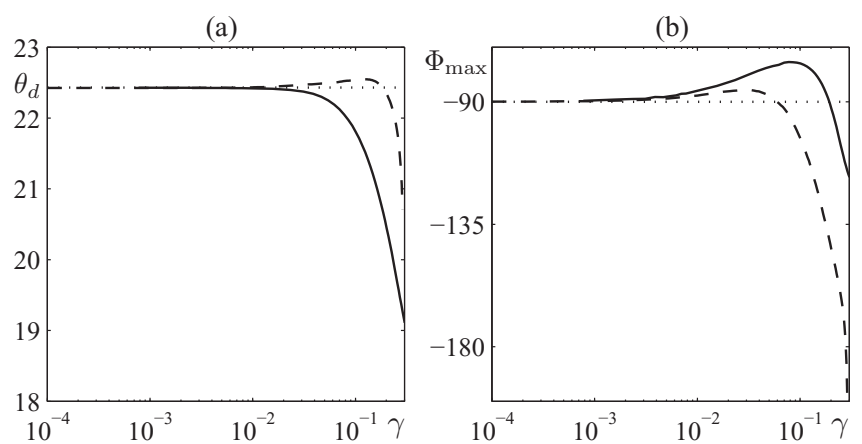

FIG. 15. Dependence on $\gamma$ of (a) resonance location $\theta_{d}$ (calculated with $\Phi=0$ ), and (b) the value $\Phi_{\max }$ which maximizes this resonance feature over $\Phi$. Solid (dashed) curves signify NS (ZV) calculations, while dotted lines show the theoretical predictions. Parameters are as in Fig. 14.

\section{E. Implications for superlattice pattern selection}

In order to understand not only how the coefficients of the amplitude equations scale with damping but also what consequences this has for the selection of superlattice patterns (or quasipatterns), one needs to know exactly how the crosscoupling coefficient $b(\theta)$ and the quadratic coefficient $\alpha$ relate to the (relative) stability of the patterns.

For a finite quadratic coefficient, $\alpha \neq 0$, superlattice patterns bifurcate subcritically and are initially unstable. A necessary condition for their stabilization at finite amplitude is [18]

$$
b_{1}+2 b_{2}<-\left|b_{4}+b_{5}+b_{6}\right|<0,
$$

where $b_{4}=b(\theta), b_{5}=b\left(\theta+\frac{2}{3} \pi\right), b_{6}=b\left(\theta+\frac{4}{3} \pi\right)$, and $b_{1}$ and $b_{2}$ are the cubic coefficients defined in Eq. (15). This condition comes from considering a set of six complex amplitude equations that describe pattern formation on a hexagonal lattice and include superlattice patterns as a solution. Examining the stability of the superlattice pattern solutions within the context of these amplitude equations then gives the eigenvalues of the superlattice patterns in terms of the coefficients of the amplitude equations. Condition (21) comes from determining possible scenarios when all eigenvalues are negative (stable).

If condition (21) is satisfied then superlattice patterns may then be stabilized for a range of parameter values, as depicted schematically in Fig. 16. Here we see that superlattice patterns are initially unstable. One eigenvalue changes sign at the saddle-node bifurcation $\mathrm{SN}_{\text {bif }}$, but it takes the switching of a second eigenvalue at the bifurcation point labeled Hex $_{\text {bif }}$ to stabilize superlattice patterns. This occurs at $\lambda_{\text {hex }}$ where

$$
\lambda_{\text {hex }}=\frac{\alpha^{2}\left[b_{1}+2 b_{2}-3\left(b_{4}+b_{5}+b_{6}\right)\right]}{4\left(b_{1}+2 b_{2}-b_{4}-b_{5}-b_{6}\right)^{2}} .
$$

Superlattice patterns are subsequently destabilized again at the point marked $\mathrm{Rh}_{\text {bif }}$ in Fig. 16. This occurs at $\lambda_{\text {rh }}$ where

$$
\lambda_{\text {rh }}=-\alpha A-\left(b_{1}+2 b_{2}+b_{4}+b_{5}+b_{6}\right),
$$

and $A$ satisfies

$$
\begin{aligned}
& {\left[2\left(b_{1}-b_{2}\right)^{2}-\left(b_{4}-b_{5}\right)^{2}-\left(b_{4}-b_{6}\right)^{2}-\left(b_{5}-b_{6}\right)^{2}\right] A^{2}} \\
& \quad-4 \alpha\left(b_{1}-b_{2}\right) A+2 \alpha^{2}=0 .
\end{aligned}
$$

The results in Eqs. (21)-(23) can be deduced from [9]. 


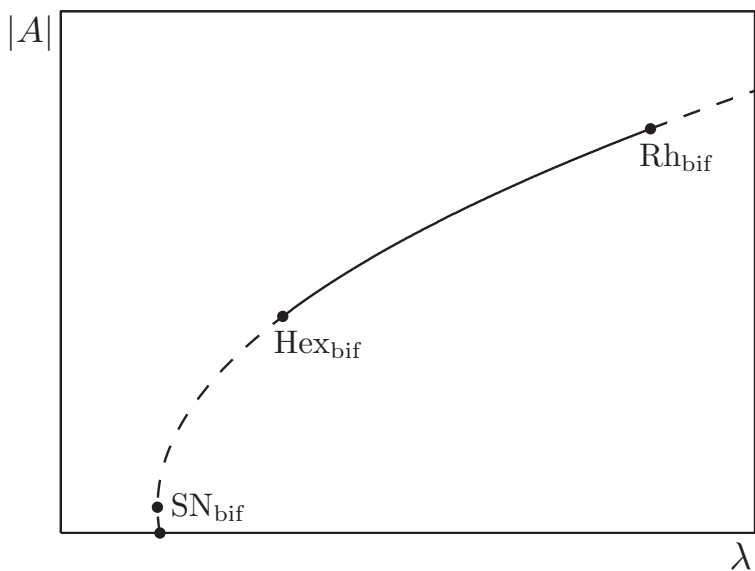

FIG. 16. Sketch of typical bifurcation diagram for a branch of superlattice patterns (measured by their amplitude $|A|$ ) showing the interval of stability $\lambda_{\text {hex }}<\lambda<\lambda_{\text {rh }}$.

The dependence of $b_{1}-b_{6}$ on $\theta$ and $\gamma$ was calculated from the NS equations for $m: n=6: 7, \chi=55^{\circ}, \phi_{m}=\phi_{n}=0$ and the same dimensionless parameters, $G_{0}=0.0396, \Gamma_{0}=$ 0.2104 , chosen for many other results presented in this paper. At low viscosity, the stability criterion, Eq. (21), is met for a large range of $\theta$ values, suggesting that numerous superlattice patterns are stable for at least some range of parameters. This is illustrated in Fig. 17(a), for 6:7 forcing and $\gamma=0.01$, where the region of stable superlattice patterns is shown as a function of the resonant angle $\theta$. This also demonstrates again the fact that the "favored" superlattice pattern (i.e., the superlattice pattern one would first expect to see experimentally) is associated with $\theta_{d}$, the angle of the resonance feature in the $b(\theta)$ curve. This particular superlattice pattern is the one that onsets first and persists over the largest interval of $a-a_{c}$. The amplitude equations derived in [9] that include superlattice patterns as a solution are variational and, consequently, a Lyapunov function can be constructed for them. This energy function is listed in Table 4.2 of [14]. The superlattice pattern with $\theta=\theta_{d}$ is not only the one that onsets
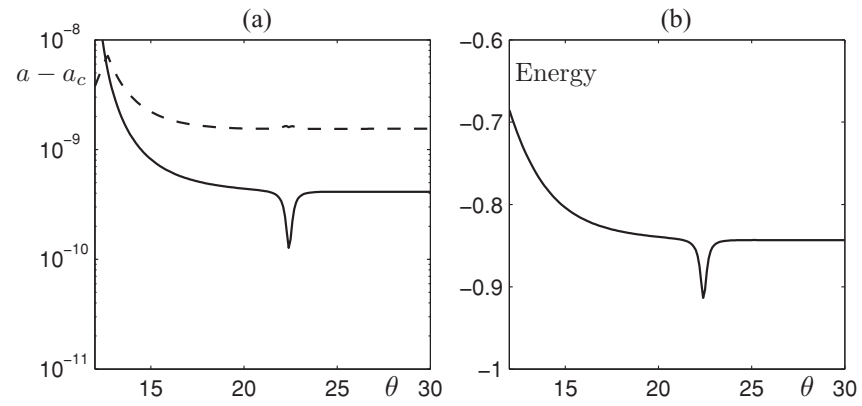

FIG. 17. (a) Bifurcation lines representing $\mathrm{Hex}_{\text {bif }}$ (solid line) and $\mathrm{Rh}_{\text {bif }}$ (dashed line) as a function of $\theta$ for $\gamma=0.01$ and other parameters as in Fig. 3. Superlattice patterns are only stable between these two lines. Those with $\theta=\theta_{d}=22.4^{\circ}$ bifurcate first with increasing $a-a_{c}$. (b) Energy of superlattice patterns as a function of $\theta$ for $a-a_{c}=5 \times 10^{-10}, \gamma=0.01$. Superlattice patterns with $\theta=\theta_{d}=22.4^{\circ}$ have lowest energy.

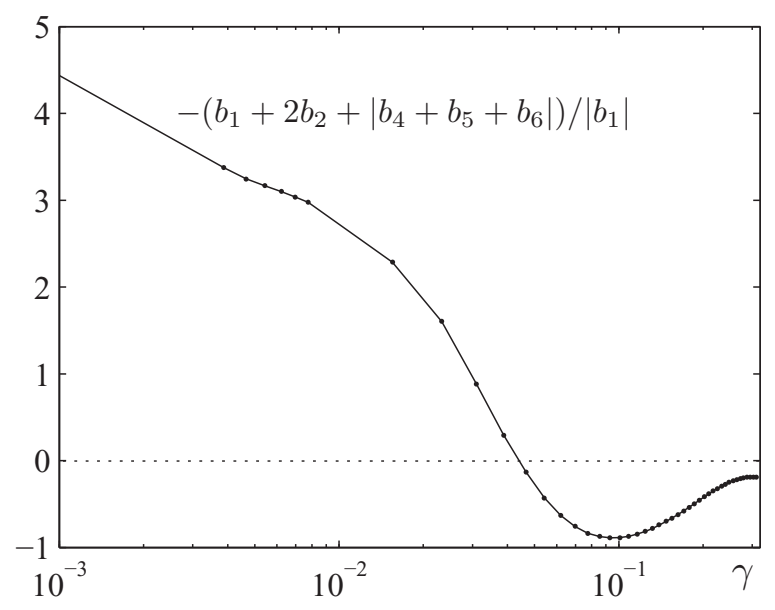

FIG. 18. The value of $-\left(b_{1}+2 b_{2}+\left|b_{4}+b_{5}+b_{6}\right|\right) /\left|b_{1}\right|$ calculated at $\theta=\theta_{d}$ as a function of $\gamma$ with parameters as in Fig. 3. This combination of coefficients must be positive if superlattice patterns are to be stabilized.

first, but is also the one that is energetically the most likely, as shown in Fig. 17(b).

For increasing but small viscosity one would expect Eq. (21) to remain satisfied, as all the cubic coefficients are proportional to $\gamma$. However, this scaling eventually fails for moderate-tolarge values of $\gamma$ and for higher viscosity the deviation from the predicted $\gamma$ scaling can lead to a change in sign of $b_{1}+$ $2 b_{2}+\left|b_{4}+b_{5}+b_{6}\right|$, as shown in Fig. 18, prohibiting stable superlattice patterns. For moderate values of $\gamma$, Eq. (21) is only satisfied for mixing angles $\chi$ near the bicritical point-and for superlattice patterns with the appropriate angle. At higher values of $\gamma$, this stability criterion cannot be satisfied at all.

At first this seems to suggest that it should be low viscosity fluids rather than moderate-to-high viscosity fluids that would be most conducive to superlattice patterns. However, for low

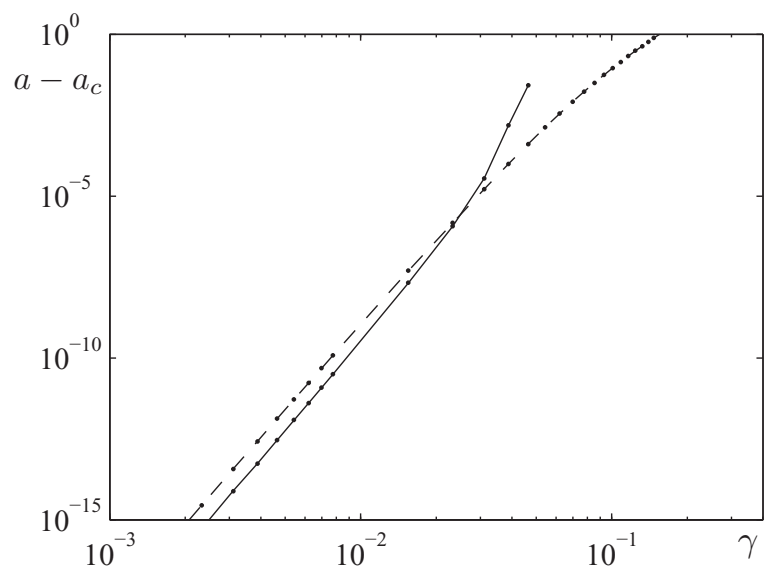

FIG. 19. Bifurcation sets for Hex $\mathrm{bif}_{\text {bif }}$ (solid line) and $\mathrm{Rh}_{\text {bif }}$ (dashed line) as a function of $\gamma$ with remaining parameters as in Fig. 3 . Superlattice patterns are only stable between these two lines when Hex $_{\text {bif }}$ occurs first [ $\gamma \lesssim 0.025$, a region in which Eq. (21) is satisfied]. Note that the vertical axis is in terms of reduced acceleration $a-a_{c}$. For each value of $\gamma, \theta=\theta_{d}$ has been used to evaluate the position of the bifurcation lines, as this is the value that gives the largest region of stable superlattice patterns; see Fig. 17. 
viscosity fluids, the region in parameter space in which they occur is tiny: Since for low viscosity $b(\theta)$ scales as $\gamma$ and $\alpha$ scales as $\gamma^{5}$, the width of the region of superlattices scales as $\gamma^{9}$, as shown in Fig. 19. So, on the one hand, at low viscosity, superlattice patterns are stable but only in a tiny (experimentally unrealistic) region of parameter space. On the other hand, if the viscosity is too large $(\gamma>0.05)$, the superlattice patterns are necessarily unstable because Eq. (21) is not satisfied.

One could question the extent to which the position of secondary bifurcations in a weakly nonlinear analysis can be expected to give a meaningful prediction for the stability regions of nonlinear patterns, but since $\alpha$ is small and all the bifurcations approach the origin as $\alpha \mapsto 0$, the distance above onset must also be small and reasonable correspondence can be expected between the calculated regions and those seen in the experiment.

\section{CONCLUSION}

A comparison of calculations from the NS and ZV equations with theory suggests that there are broadly three regions of behavior delineated by the value of the damping parameter $\gamma$. Firstly, for $\gamma<0.01$, there is excellent quantitative agreement between the NS equation, the $\mathrm{ZV}$ equation, and the theoretical predictions in $[11,12]$. This is the region where single-frequency resonant features are most prominent and is the region where most single-frequency experiments have been performed.

Secondly, there is a regime of moderate damping from $\gamma \approx 0.01$ to $\gamma \approx 0.1$ where there remains a fair degree of qualitative agreement with theory. The location of key resonances, for example, can be predicted with reasonable accuracy, and increasing viscosity will increase the size of the resonant feature located there. A notable exception is the phase $(\Phi)$ dependence, which tends to deviate more from predicted values than the other scaling laws over this range. Furthermore, the single-frequency 1:2 resonant feature is no longer apparent in the $b(\theta)$ curve. It is interesting to note that the ZV model continues to agree well with the NS equation in this regime.

Finally, there is a high viscosity regime for $\gamma>0.1$ where there is generally not even qualitative agreement between the NS equations and the theory of [12]. Increasing $\gamma$ in this regime, for example, could cause a decrease in $b\left(\theta_{d}\right)$ rather than an increase. Some resonances may switch from enhancing to suppressing or become washed out entirely. In this regime, there is qualitative, but not quantitative agreement between the $\mathrm{NS}$ and the ZV equations.

The deviation from the scaling predicted by the low viscosity symmetry-based theory has a significant impact on pattern selection. In Sec. III E it was shown that the deviation from the predicted scaling led to an optimal range of $\gamma$ for experiments that aim to find superlattice patterns: large enough to benefit from an increase in resonance strength with $\gamma$ and an increase in the range of forcing values yielding stable patterns, but not so large that the predicted behavior is overwhelmed by new (in this case, suppressing) effects. It is perhaps no coincidence that, although some multifrequency experiments have been carried out for high viscosities $(\gamma>0.5)$ [6], no superlattice patterns have been
TABLE I. Typical values for the physical parameters as quoted in experimental papers and the corresponding values for $k_{0}$, as calculated from Eq. (1), and $\gamma=2 \nu k_{0}^{2} / m \omega$. An asterisk marks values that were not given explicitly in the experimental papers, but are typical for the fluid used.

\begin{tabular}{llcccccc}
\hline \hline & $\begin{array}{c}\sigma \\
\text { Ref. }\end{array}$ & $\begin{array}{c}\rho \\
(\mathrm{dyn} / \mathrm{cm})\end{array}$ & $\begin{array}{c}v \\
\left(\mathrm{~g} / \mathrm{s}^{3}\right)\end{array}$ & $\begin{array}{c}\mathrm{cSt}) \\
m: n\end{array}$ & $\begin{array}{c}m \omega \\
(\mathrm{Hz})\end{array}$ & $\begin{array}{c}k_{0} \\
(1 / \mathrm{cm})\end{array}$ & $\gamma$ \\
\hline$[2]$ & 18.3 & 0.892 & 3.4 & 1 & 27 & 4.89 & 0.010 \\
{$[2]$} & 18.3 & 0.892 & 3.4 & 1 & 45 & 8.32 & 0.017 \\
{$[3]$} & 19.4 & 0.920 & 5 & 1 & 10 & 0.99 & 0.0015 \\
{$[25]$} & 19.8 & 0.929 & 8.9 & 1 & 10 & 0.99 & 0.0028 \\
{$[5]$} & $20.6^{*}$ & $0.95^{*}$ & 20 & $4: 5$ & 88 & 14.2 & 0.15 \\
{$[5]$} & $20.6^{*}$ & $0.95^{*}$ & 20 & $6: 7$ & 98.64 & 15.5 & 0.16 \\
{$[6]$} & 20.6 & 0.95 & 23 & $2: 3$ & 50 & 9.00 & 0.12 \\
{$[6]$} & $20.6^{*}$ & $0.95^{*}$ & 47 & $2: 3$ & 50 & 9.00 & 0.24 \\
{$[7]$} & $20.6^{*}$ & $0.95^{*}$ & 20 & $4: 5$ & 24 & 4.18 & 0.046 \\
\hline \hline
\end{tabular}

reported there. In fact, most two-frequency experiments have been carried out for $\gamma \approx 0.1-0.3$ (Typical values for the various physical parameters used in key experimental papers and the corresponding values for $\gamma$ are shown in Table I). In the example shown in Fig. 4, this is in the region of $\gamma$ where the $b(\theta)$ curve achieves its maximum value. Figure 19 suggests that for these values of $\gamma$ there is no region of stable superlattice patterns. However, this theoretical result does not contradict the experiments of [5] because, although the remaining fluid parameters are comparable, the results shown in Fig. 19 are for $\left(f_{6}, f_{7}\right)=a G_{0}(\cos \chi, \sin \chi)$ with $\chi=55^{\circ}$, while the experiments show a small region of superlattice patterns only for values of $\chi$ of approximately $59^{\circ}$ and above. A more detailed comparison with the experiments of [5] is undertaken in [14].

We also found that, just as there is an optimal range of the damping parameter $\gamma$ for locating certain patterns such as superlattices, there is an optimal phase $\Phi$ as well. For the moderate values of $\gamma$ used in the relevant experiments, this optimal phase can be deduced from the NS equation and/or from the $\mathrm{ZV}$ equation but it is not captured as well by the low damping symmetry-based theory as other scaling laws. This could explain some of the discrepancy between theory and experiment found in [11].

The results in this paper further underline the fact that in the Faraday problem the nonlinear pattern selection process depends strongly on which resonant triad interactions can occur and this, in turn, can be deduced from the linear problem, which determines the resonance angle $\theta_{d}$. In Sec. III B we derived a method for estimating the position of $\theta_{d}$ from the linear stability curves of the $\mathrm{ZV}$ equation that also provided a good estimate of the position of $\theta_{d}$ for the NS equation, even for the moderate values of the viscosity that were considered.

\section{ACKNOWLEDGMENTS}

The work of J.P. was supported by Spanish Ministerio de Educación y Ciencia (Project No. ESP2007-65221) and Ministerio de Ciencia e Innovación (Project No. AYA201019081). 
[1] M. Faraday, Philos. Trans. R. Soc. London 121, 299 (1831).

[2] D. Binks and W. van de Water, Phys. Rev. Lett. 78, 4043 (1997).

[3] C. Wagner, H.-W. Müller, and K. Knorr, Phys. Rev. E 68, 066204 (2003).

[4] W. Edwards and S. Fauve, J. Fluid Mech. 278, 123 (1994).

[5] A. Kudrolli, B. Pier, and J. Gollub, Physica D 123, 99 (1998).

[6] H. Arbell and J. Fineberg, Phys. Rev. Lett. 84, 654 (2000).

[7] T. Epstein and J. Fineberg, Phys. Rev. E 73, 055302 (2006).

[8] Y. Ding and P. Umbanhowar, Phys. Rev. E 73, 046305 (2006).

[9] B. Dionne, M. Silber, and A. C. Skeldon, Nonlinearity 10, 321 (1997).

[10] M. Silber and A. C. Skeldon, Phys. Rev. E 59, 5446 (1999).

[11] J. Porter and M. Silber, Phys. Rev. Lett. 89, 084501 (2002).

[12] C. Topaz, J. Porter, and M. Silber, Phys. Rev. E 70, 066206 (2004).

[13] W. Zhang and J. Viñals, J. Fluid Mech. 336, 301 (1997).

[14] A. C. Skeldon and G. Guidoboni, SIAM J Appl. Math. 67, 1064 (2007).
[15] T. B. Benjamin and F. Ursell, Proc. R. Soc. London A 225, 505 (1954).

[16] J. Porter and M. Silber, Physica D 190, 93 (2004).

[17] T. Besson, W. S. Edwards, and L. S. Tuckerman, Phys. Rev. E 54, 507 (1996).

[18] M. Silber, C. Topaz, and A. C. Skeldon, Physica D 143, 205 (2000).

[19] A. M. Rucklidge and M. Silber, Phys. Rev. E 75, 055203(R) (2007).

[20] A. M. Rucklidge and W. Rucklidge, Physica D 178, 62 (2003).

[21] W. Zhang and J. Viñals, Phys. Rev. E 53, 4283 (1996).

[22] J. Porter, C. M. Topaz, and M. Silber, Phys. Rev. Lett. 93, 034502 (2004).

[23] L. Trefethen, Spectral Methods in MATLAB (SIAM, Philadelphia, 2000).

[24] D. Jordan and P. Smith, Nonlinear Ordinary Differential Equations (Oxford University Press, Oxford, 1987).

[25] H. W. Müller, H. Wittmer, C. Wagner, J. Albers, and K. Knorr, Phys. Rev. Lett. 78, 2357 (1997). 